\title{
Marker profiles of human endometrial stem cells at various passages cultured in-vitro
}

\begin{abstract}
Endometrium is a dynamic organ, which undergoes extensive proliferation and regeneration throughout reproductive cycle of women. Hence, extensive research on adult stem cells of endometrium is underway. An easy approach to identify stem cells is its marker characterization. However, does these markers behave same in all conditions of stem cells in-vitro is uncertain? Hence, the main objective of the study is to check if mesenchymal stem cells of the endometrium (eMSCs) could retain its marker characterization at various passages in-vitro.
\end{abstract}

The study design was accomplished as follows: The endometrial tissue $(n=10)$ were collected from reproductively active women with a mean age of $35 \pm 1.5$ and body mass index of $24 \pm 1.4$, undergoing D\&C/hysterectomy. Samples were collected in a sterile manner. The plastic, adherent mesenchymal stem cells obtained from endometrial tissue (eMSCs) are characterized for markers such as CD44, CD166, CD106, CD49d, CD31, CD54, CD34, CD117, CD90, CD105, CD73, CD140b, ABCG2 at various passages (P1, P3, P5 \& P10) in-vitro using flowcytometry. The obtained results were analysed statistically using student's t-test and significant results are discussed.

The study identified tissue-specific markers of eMSCs, where the percentage expression of most of the markers was consistently similar at P3 and P10, expect for CD49d and CD54, which showed a reduction pattern and ABCG2, which showed an increase in percentage at P10 as compared to P3. This article reveals the significance of in-depth marker characterization of human endometrial stem cells in-vitro.
Volume 2 Issue I - 2017

Pankaj Kaingade, ${ }^{1,2}$ Amar Nikam, ${ }^{2}$ Sachin

Kulkarni, ${ }^{2}$ Indumathi Somasundaram ${ }^{3}$

'Department of Biochemistry, Charotar University of Science and Technology, India

2Department of Obstetrics and Gynecology, Pristine Women's Hospital, India

${ }^{3}$ Department of Stem cells and Regenerative Medicine, DY Patil University, India

Correspondence: Indumathi Somasundaram, Department of Stem cells and Regenerative Medicine, DY Patil University, Kolhapur, Maharashtra, India, Tel +918520802540

Email industemcell0I@gmail.com

Received: December 05, 2016 | Published: January II, 2017

\section{Introduction}

Endometrium, the inner lining of the uterus, plays an integral role throughout reproductive life of every woman. ${ }^{1}$ It undergoes cyclic changes to proliferate and regenerate. It serves as an optimal environment for blastocyst implantation and as a site for placental generation. ${ }^{2}$ Like any other organ in the human body, endometrium is susceptible to various disorders such as endometriosis, endometrial hyperplasia, endometritis, to name a few. ${ }^{3-5}$ Being a significant component of the uterus, it is essential to safeguard endometrium for a healthy womanhood. The key factor that stays within endometrium which regulates these aforesaid functions is stem cells. This is even more precisely proven by the fact that endocrine mediated menstrual cycle involves the shedding of the inner lining of the uterus and its subsequent regeneration effectively by means of stem cells. ${ }^{6}$ Hence, identification of stem cells and its potent role in physiology and pathology of endometrium is imperative and are underway.

In this regard, one essential attribute that identifies stem cells is its marker characterization. Although several markers have been identified in stem cells of endometrium, including our previously published paper, ${ }^{6,7}$ there are no current literatures to check its marker characterization at various passages cultured in-vitro. It is understood from the literatures that mesenchymal stem cells are potent source of wound healing and repair mechanisms. ${ }^{8,9}$ Thus tissue-specific markers of mesenchymal stem cells at cultured conditions are important to characterize. Hence, we, herein, characterize some markers of endometrium derived mesenchymal stem cells, at various passages (P1, P3, P5 \& P10) in-vitro.

\section{Materials and methods}

\section{Subjects}

The study was approved by the Institutional Ethics Committee of Pristine Women's Hospital (Kolhapur, Maharashtra, India). The participants were informed about the purpose of sampling and written informed consent of the patients was obtained prior to sampling. All samples were transported to the laboratory in a sterile container. Human endometrial biopsies were collected from non-malignant, reproductively active women $(n=10)$ with a mean age of $35 \pm 1.5$ and body mass index of $24 \pm 1.4$, undergoing D\&C/hysterectomy. The patients had not undergone exogenous hormonal treatment for at least 3 months prior to surgery. All samples were processed within $2 \mathrm{hr}$ of collection.

\section{Isolation and culturing of endometrial cells}

The samples were isolated according to Indumathi et al. ${ }^{7}$ The samples were collected and transported to laboratory by aseptic technique. The tissue samples were washed in phosphate-buffered saline to remove blood and other debris. The washed samples were then minced mechanically and treated with trypsin for adequate cell separation. The cells were obtained by centrifugation. The cells were cultured in tissue culture flasks with DMEM supplemented with $10 \%$ hUCBS. The cultures were maintained in $\mathrm{CO}_{2}$ incubator containing $5 \% \mathrm{CO}_{2}, 95 \%$ humidity at $37^{\circ} \mathrm{C}$. Medium was changed every $48 \mathrm{hr}$ and replenished with DMEM supplemented with 10\% hUCBS. At $80 \%$ confluency, cells were trypsinized and passaged further. 


\section{Marker characterization}

Immunophenotypic characterization was performed by Fluroscence-Activated Cell Sorter (FACS) in a BD (Becton, Dickinson) FACS Aria. Fluorescence excitation was carried out by using an argon-ion and a red LASER of $488 \mathrm{~nm}$ and $632 \mathrm{~nm}$ respectively. The fluorescence emission was collected by using corresponding detectors. Approximately $1 \times 105$ cells were stained with predefined antibody cocktails (Table 1 ). The stained cells were incubated in the dark for $20 \mathrm{~min}$ at room temperature, washed with wash flow buffer (BD Biosciences), resuspended in PBS and then characterized in a BD FACS Aria. Data acquisition and analysis were accomplished by using BD FACS Diva software (BD Biosciences). A minimum of 10,000 events were characterized and recorded.

Table I Cell markers used. Detailed antibody list together with the relevant flurochrome (PE phycoerythrin, FITC fluorescein isothiocyanate, APC allophycocyanin, PERCP peridinin chlorophyll protein)

\begin{tabular}{lll}
\hline Sr. no. & Markers & Flurochrome \\
\hline 1 & CD44 & FITC (BD Bioscience) \\
2 & CDI66 & PE (BD Bioscience) \\
3 & CDI06 & FITC (BD Bioscience) \\
4 & CD49d & PE (BD Bioscience) \\
5 & CD31 & FITC (BD Bioscience) \\
6 & CD54 & PERCP (BD Bioscience) \\
7 & CD34 & PE (BD Bioscience) \\
8 & CDII7 & APC (e- Biosciences) \\
9 & CD90 & PERCP (e- Biosciences) \\
10 & CDI05 & APC (e- Biosciences) \\
11 & CD73 & PE (BD Bioscience) \\
12 & CDI40b & PE (BD Bioscience) \\
13 & ABCG2 & PE (e- Biosciences) \\
\hline
\end{tabular}

\section{Statistical analysis}

All data obtained from endometrium samples $(n=10)$ is presented as mean \pm SEM. Statistical analysis was carried out by using the software, Statistical Package for Social Science, SPSS 20.0 (SPSS, Chicago, III., USA). The data were analyzed by using Student's t-test and P-values were calculated to determine the statistically significant variations. Results were considered statistically significant when $\mathrm{p}<0.05^{*}$.

\section{Results}

The schematic work flow of the study has been illustrated, where the endometrial derived mesenchymal stem cells (eMSCs) at various passages (P1, P3, P5 \&P10) were phenotypically characterized for HSC, MSC, Cell adhesion molecules and other markers (Figure 1). The flowcytometric illustrations of these markers at P3 and P10 were represented (Figures $2 \& 3$ ). Overall, the percentage expression of most of the markers was consistently similar at P3 and P10, expect for $\mathrm{CD} 49 \mathrm{~d}$ and $\mathrm{CD} 54$, which showed a reduction pattern and $\mathrm{ABCG} 2$, which showed an increase in percentage at P10 as compared to P3 (Figure 4). Its marker characteristics of various populations are specified in the form of graph: HSC/ MSC (Figure 4A), other markers (Figure 4B) and cell adhesion molecules (Figure 4C). Furthermore, individual marker expression profile of all samples $(n=10)$ at various passages P1, P3, P5 and P10 are also specified (Supplementary Tables 3-6).

A remarkable expression (90-100\%) of mesenchymal stem cell markers such as CD90, CD105 and CD73 were observed in both earlier and later passages and thereby confirming its presence as MSCs even at P10 conditions. Additionally, certain cell adhesion molecules such as CD49d, CD44, CD54 and CD166 also exhibited remarkable expression even at P10, with slight decrease in percentage at P10. Certain non-mesenchymal, hematopoietic/endothelial markers such as CD34, CD31 and CD106 showed sparse expression confirming its homogeneity. Besides, CD117, a stem cell factor C-KIT marker and CD140b, a PDGFRb receptor was also remarkable throughout the culture.

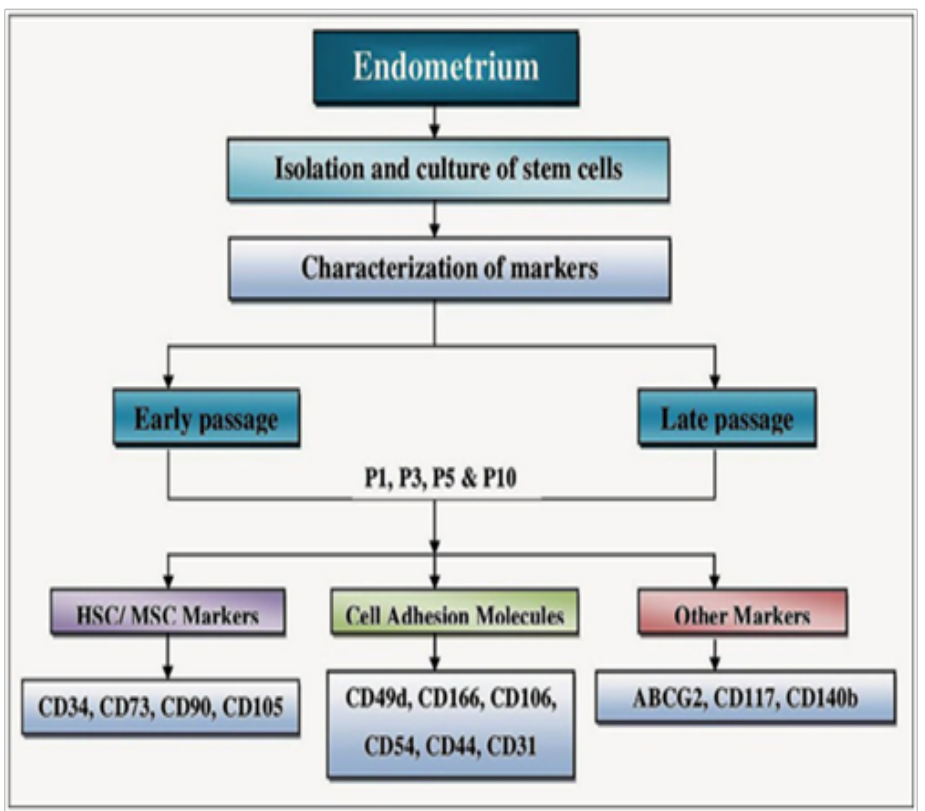

Figure I Schematic representation of the study. 

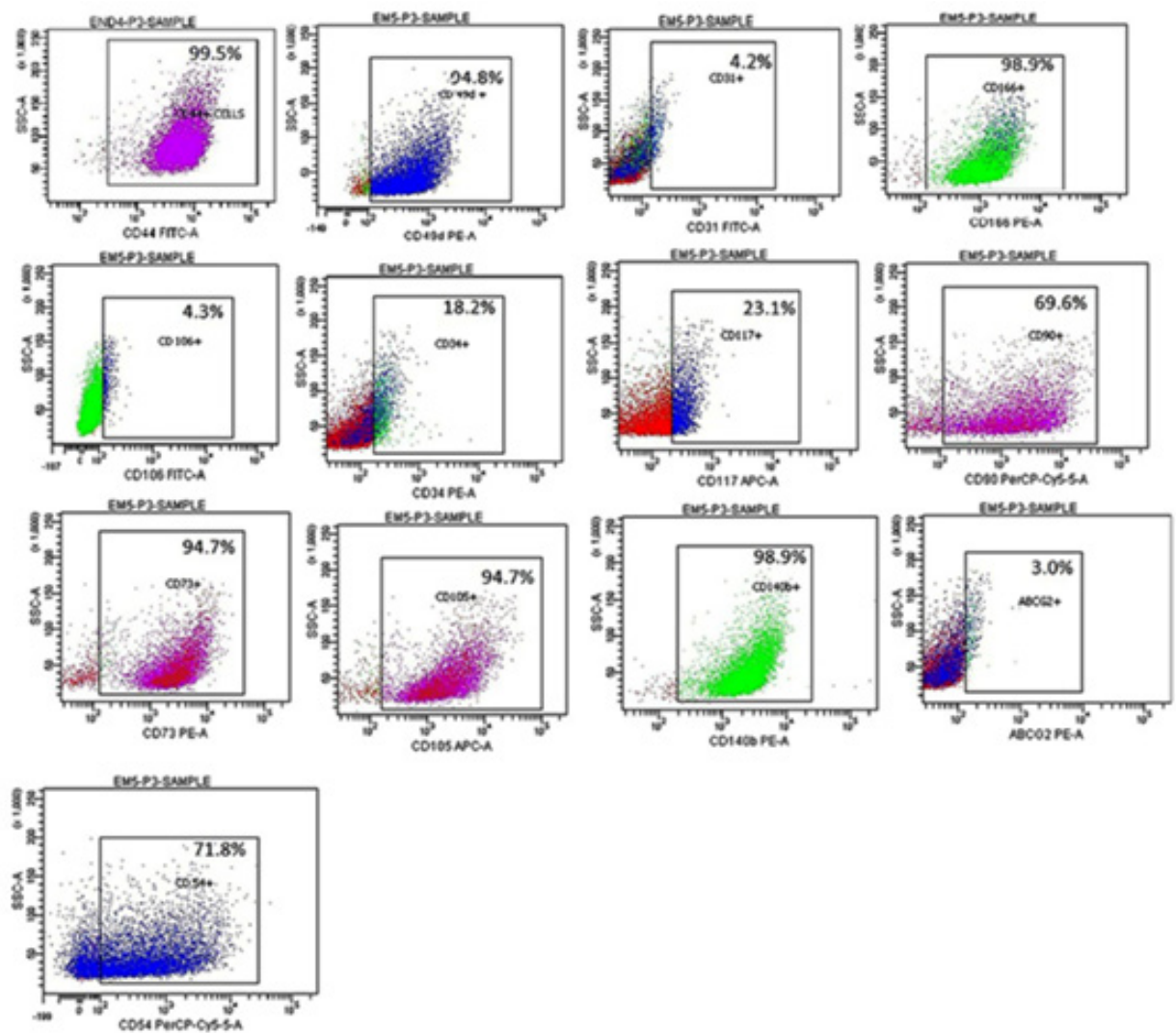

Figure 2 Marker profiles characterized by flowcytometry at P3.
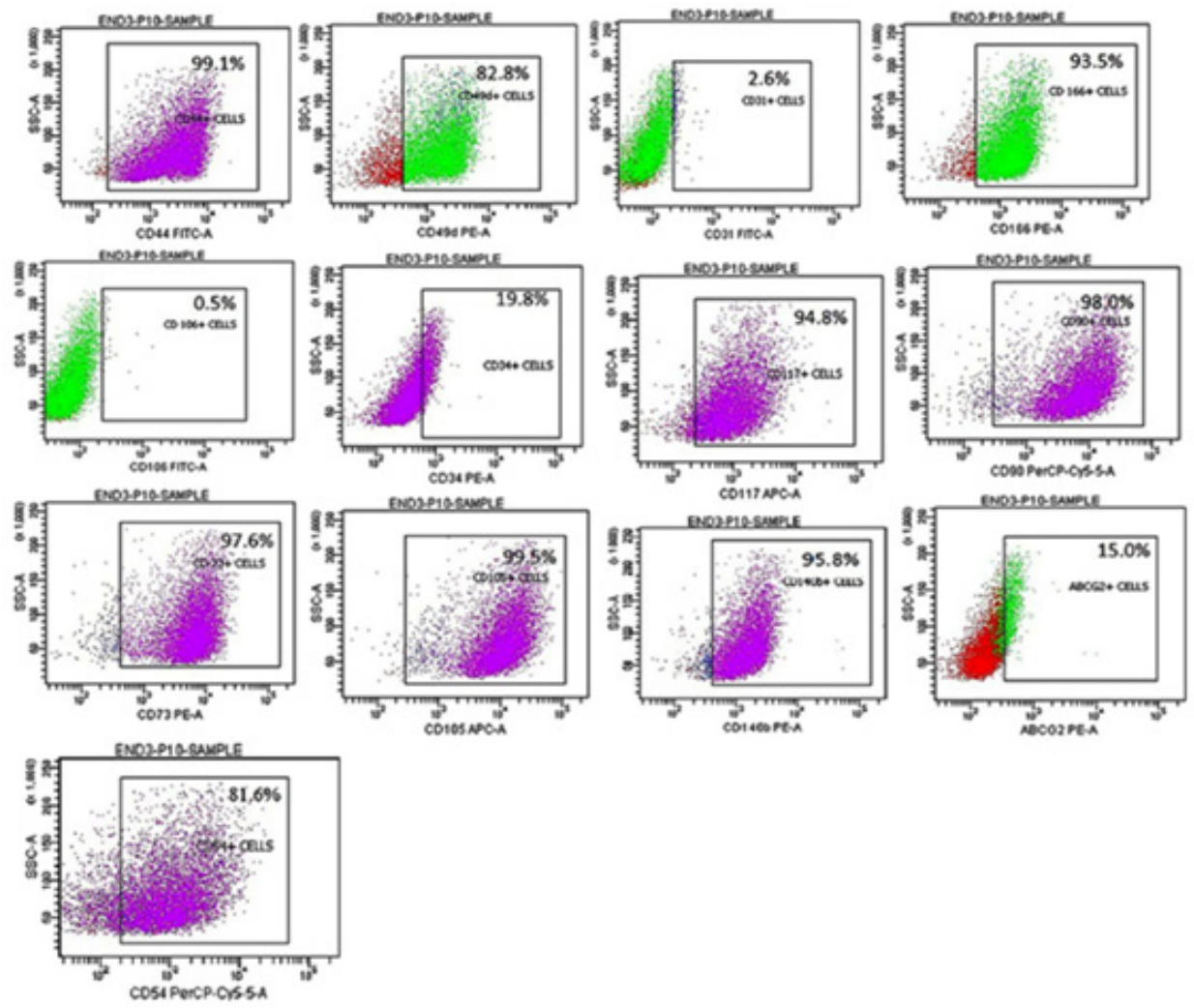

Figure 3 Marker profiles characterized by flowcytometry at PI0. 


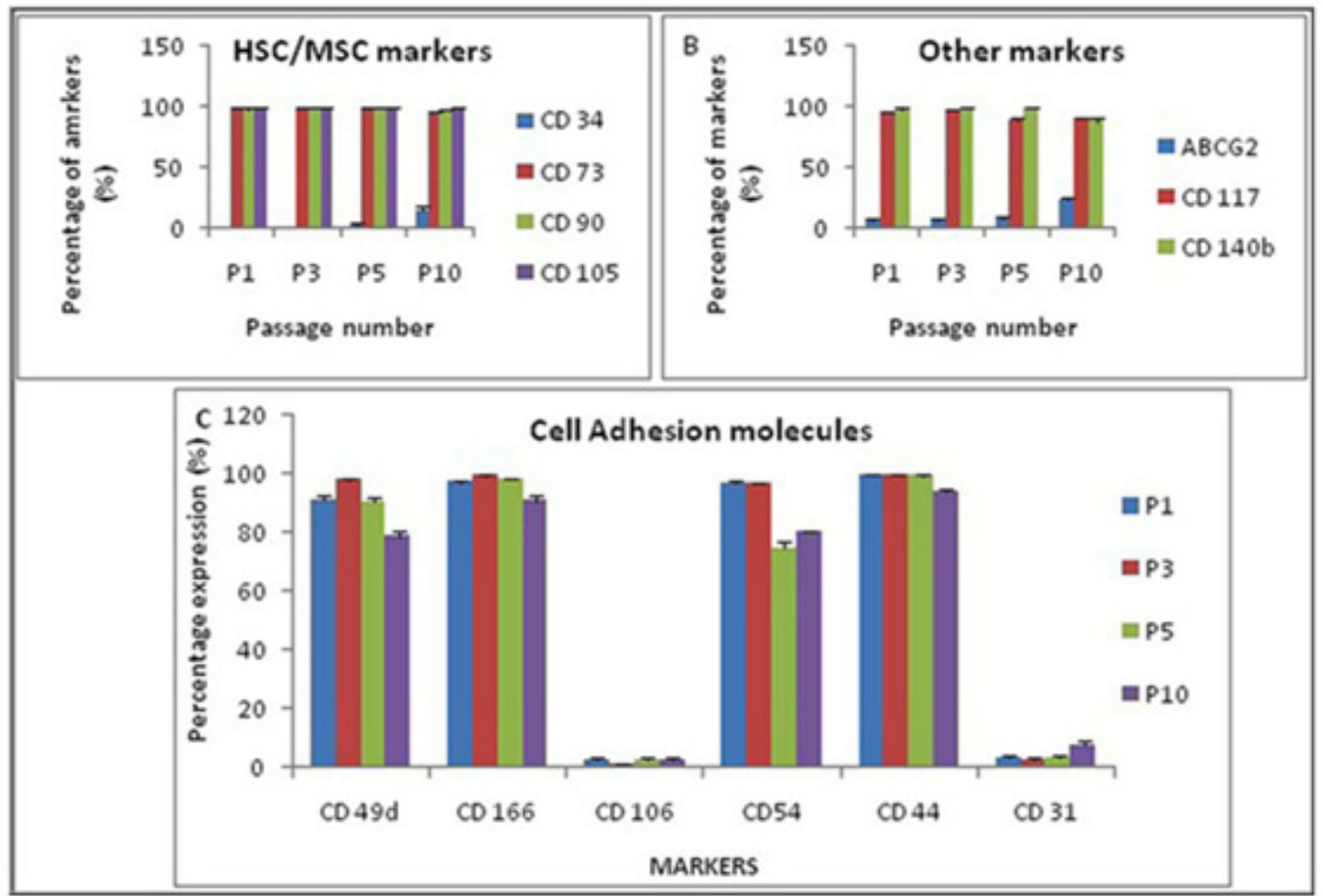

Figure 4A Marker profile comparison at P3 and PIO of HSC/MSC markers.

Figure 4B Marker profile comparison at P3 and PIO of other markers.

Figure 4C Marker profile comparison at P3 and PIO of cell adhesion molecules markers.

The values are represented in the form of means \pm SEM. $P<0.05$ are represented as*

Table 2 Expression profile of cell surface markers. The values are represented in the form of means \pm SEM

\begin{tabular}{|c|c|c|}
\hline Markers & Endometrium early passage (P3) & Endometrium late passage $(\mathrm{P} \mid 0)$ \\
\hline \multicolumn{3}{|c|}{ Cell adhesion molecules } \\
\hline CD44 & $99.37 \pm 0.13$ & $93.76 \pm 1.22$ \\
\hline CD49d & $97.87 \pm 0.68$ & $78.6 \pm 1.55$ \\
\hline CD3। & $2.92 \pm 0.53$ & $7.88 \pm 1.18$ \\
\hline CD54 & $96.63 \pm 0.42$ & $80.18 \pm 0.55$ \\
\hline CDI06 & $1.15 \pm 0.15$ & $2.59 \pm 0.64$ \\
\hline \multicolumn{3}{|c|}{ Hematopoietic \& C-kit marker } \\
\hline CD34 & $0.8 I \pm 0.1$ & $15.4 \pm 2.23$ \\
\hline CD90 & $99.06 \pm 0.22$ & $97.15 \pm 0.30$ \\
\hline CD73 & $99.64 \pm 0.03$ & $96.51 \pm 0.32$ \\
\hline CDI05 & $99.67 \pm 0.09$ & $99.24 \pm 0.09$ \\
\hline \multicolumn{3}{|c|}{ Perivascular markers } \\
\hline CDI40b & $99.12 \pm 0.12$ & $88.18 \pm 2.28$ \\
\hline \multicolumn{3}{|c|}{ Side population markers } \\
\hline$A B C G 2$ & $6.2 \pm 0.87$ & $22.43 \pm 2.02$ \\
\hline
\end{tabular}




\section{Discussion}

Endometrium is a vibrant tissue indulged in more than 400 cycles of regeneration and differentiation which is highly crucial during the reproductive period of women. ${ }^{1,2,6}$ It bears a dynamic ability to generate an endometrial layer of $5-7 \mathrm{~mm}$ thickness following menstruation. It has been previously demonstrated that the existence of stem cell population is responsible for the regeneration during the menstrual cycle. ${ }^{10}$ Thus, identifying the tissue-specific stem cell markers in normal endometrium paves way to identify the same in disease condition. Our study carried out a marker profiling of endometrial cells in heterogenous fresh isolates. ${ }^{7}$ However, as reported in several literatures, evaluating homogenous MSCs is equally important for ascertaining its utility in wound healing and repair mechanisms. This formed the basis of our work. Besides, many studies had carried out cultures in fetal bovine serum (FBS). We in this study cultured and characterized the MSCs supplemented with human umbilical cord serum (hUCBS), for a cost-effective work. From the results, we prefer that hUCBs could be used as an alternative for FBS in further culture and characterization of eMSCs, or any MSCs. The growth factor secreted from hUCB serum also supports our statement. ${ }^{11}$ Besides, this was also supported by Shetty who demonstrates hUCB as an alternative source for FBS. ${ }^{12}$

The positive antigenic expressions of CD73, CD90 and CD105 were previously confirmed in endometrial derived mesenchymal stem cells. ${ }^{13}$ Besides, endometrial stem cells have been previously evaluated for an array of surface markers which include hematopoietic markers (CD 34, HLA-DR) (Table 2), mesenchyme markers (CD73, CD90 and CD105), Cell adhesion molecules (CAM) (CD44, CD31), pluripotent marker: SSEA-4 and other unique markers like ABCG2, CD140b and CD117. ${ }^{13-16}$ Our results were coherent with all the above results in our studied marker panels even at passage 10. In this study along with the aforesaid markers we focus on certain other markers: Intercellular adhesion molecule -1(ICAM-1)/ CD54, a calcium independent cell adhesion molecule which was found positive in mesenchymal stem cells derived from placenta and bone marrow, ${ }^{17,18}$ activated leukocyte cell adhesion molecule (ALCAM)/CD 166, yet another cell adhesion molecule which was found to be expressed by mesenchymal stem cells. ${ }^{19}$ Besides, Platelet derived growth factor-receptor $\beta$ (PDGF-R $\beta / \mathrm{CD} 140 \mathrm{~b}$ ), a perivascular marker ${ }^{20}$ is known to be expressed by colony-forming endometrial stromal cells, ${ }^{10}$ was remarkable in our study as well throughout the passages until P10. This seems to be an important growth factor of endometrial stromal cells in activating many key self renewal pathways. PDGF-R $\beta / C D 140 b$, identified as a pericyte marker, along with CD146+ cells, by Schwab \& her coworkers, ${ }^{6}$ seems to be an important component in identifying and isolating MSCs as well. As CD140b turns to be highly remarkable in our cultures, we will move a step ahead to sort out CD140b+CD146+ cells, to find the perivascular nature of these MSCs and carry out an angiogenic profiling so as to identify its implications in vascular diseases.

There are several markers that are associated with diseases. C-kit/ CD 117 was one such marker. CD117 receptor is expressed on the surface of stem cells and other cell types. Mutations of this receptor are known to be associated with carcinomas. ${ }^{21}$ A comparative analysis of c-kit expression was done on women with and without endometriosis by Catherine Uzan et al. ${ }^{22}$ Results show that CD117 expression was higher in women with endometriosis when compared to normal women. ${ }^{22}$ This is one such marker that has been characterized in- depth under disease conditions. However, other markers are yet to be explored.

Although our present study is basic, we anticipate that, this article reveals the significance of in-depth marker characterization of human endometrial MSCs in-vitro, thereby paving way later to explore several unexplored features of endometrial characterization in diseased conditions.

\section{Acknowledgements}

None.

\section{Conflict of interest}

The author declares no conflict of interest.

\section{References}

1. Padykula HA. Regeneration in the primate uterus:the role of stem cells. Ann N Y Acad Sci. 1991;622:47-56.

2. Zenclussen AC, Hämmerling GJ. Cellular regulation of the uterine microenvironment that enables embryo implantation. Front Immunol. 2015;6:321.

3. Cervelló I, Mirantes C, Santamaria X, et al. Stem cells in human endometrium and endometrial carcinoma. Int $J$ Gynecol Pathol. 2011;30(4):317-327.

4. Figueira PG, Abrão MS, Krikun G, et al. Stem cells in endometrium and their role in the pathogenesis of endometriosis. Ann N Y Acad Sci. 2011;1221:10-17.

5. Gargett BE, Chan RW. Endometrial stem/progenitor cells and proliferative disorders of the endometrium. Minerva Ginecol. 2006;58(6):511-526.

6. Schwab K, Chan R, Gargett C. Putative stem cell activity of human endometrial epithelial and stromal cells during the menstrual cycle. Fertil Steril. 2005;84(Supp1 2):1124-1130

7. Indumathi S, Harikrishnan R, Rajkumar JS, et al. Prospective biomarkers of stem cells of human endometrium and fallopian tube compared with bone marrow. Cell Tissue Res. 2013;352(3):537-549.

8. Duscher D, Barrera J, Wong VW, et al. Stem cells in wound healing: the future of regenerative medicine? A mini-review. Gerontology. 2016;62(2):216-225.

9. Wu Y, Chen L, Scott PG, et al. Mesenchymal stem cells enhance wound healing through differentiation and angiogenesis. Stem Cells. 2007;25(10):2648-2459.

10. Mclennan CE, Rydell AH. Extent of endometrial shedding during normal menstruation. Obstet Gynecol. 1965;26(5):605-621.

11. Patki S, Patki U, Patil R, et al. Comparison of the levels of the growth factors in umbilical cord serum and human milk and its clinical significance. Cytokine. 2012;59(2):305-308.

12. Shetty P, Bharucha K, Tanavde V. Human umbilical cord blood serum can replace fetal bovine serum in the culture of mesenchymal stem cells. Cell Biol Int. 2007;31(3):293-298.

13. Gargett CE, Schwab KE, Zillwood RM, et al. Isolation and culture of epithelial progenitors and mesenchymal stem cells from human endometrium. Biol Reprod. 2009;80(6):1136-1145.

14. Meng X, Ichim TE, Zhong J, et al. Endometrial regenerative cells: a novel stem cell population. $J$ Transl Med. 2007;5:57. 
15. Dimitrov R, Timeva T, Kyurkchiev D, et al. Characterization of clonogenic stromal cells isolated from human endometrium. Reproduction. 2008;135(4):551-558.

16. Masuda H, Matsuzaki Y, Hiratsu E, et al. Stem cell-like properties of the endometrial side population: implication in endometrial regeneration. PLoS One. 2010;5(4):e10387.

17. Fukuchi Y, Nakajima H, Sugiyama D, et al. Human placenta-derived cells have mesenchymal stem/progenitor cell potential. Stem Cells. 2004;22(5):649-658.

18. Deans RJ, Moseley AB. Mesenchymal stem cells: biology and potential clinical uses. Exp Hematol. 2000;28(8):875-84.
19. Etheridge SL, Spencer GJ, Heath DJ, et al. Expression profiling and functional analysis of wnt signaling mechanisms in mesenchymal stem cells. Stem Cells. 2004;22(5):849-860.

20. Hirschi KK, D'amore PA. Pericytes in the microvasculature. Cardiovasc Res. 1996;32(4):687-698.

21. Edling CE, Hallberg B. c-Kit-a hematopoietic cell essential receptor tyrosine kinase. Int J Biochem Cell Biol. 2007;39:1995-1998.

22. Uzan C, Cortez A, Dufournet C, et al. Endometrium from women with and without endometriosis, and peritoneal, ovarian and bowel endometriosis, show different c-kit protein expression. J Reprod Immunol. 2005;65(1):55-63. 